\title{
Reversible intraventricular conduction defect in aconite poisoning: A Case report
}

\author{
AK Yadav, S Chaudhuri, PP Gupta, R Chaudhary, BD Aryal, B Sah, G Malla \\ Department of General Practice \& Emergency Medicine \\ B.P. Koirala Institute of Health Sciences, Dharan, Nepal
}

\begin{abstract}
Aconitine and related alkaloids found in the Aconitum species are highly cardiotoxic and neurotoxic. The wild plant (especially the roots and root tubers) is extremely toxic. Severe aconite poisoning can occur after accidental ingestion of the wild plant or consumption of an herbal made from aconite roots. In traditional Chinese medicine, aconite roots are used only after processing to reduce the toxic alkaloid content. Soaking and boiling during processing will hydrolyze aconite alkaloids into less toxic and non-toxic derivatives. However, the use of a larger than recommended dose and inadequate processing increases the risk of poisoning. The aconite herb is one of the commonly-prescribed ingredients for various clinical problems. However, due to its narrow therapeutic index, toxicities are not uncommonly encountered, including life-threatening cardiac arrhythmias like ventricular arrhythmias.
\end{abstract}

Keywords: Aconitine poisoning, intraventricular conduction defect, right bundle branch block, ventricle premature complexes

\section{Introduction}

Aconitum ferox is the species found in Nepal, India, and the Himalaya Mountains. An extract of

this plant has been used as an antipyretic in Ayurvedic medicine, after detoxification ${ }^{1}$. Aconite tubers were among the most toxic plants known but it had been used in the eastern and western therapeutics for

\section{Address for correspondence}

Dr. Ajay Kumar Yadav

Assistant Professor

Department of GP and EM

B.P. Koirala Institute of Health Sciences, Dharan

Email: ajay.yadav@bpkihs.edu centuries. The root was used to poison the hunting spikes ${ }^{2}$. In annonated check list of flowering plants of Nepal, 38 Aconitum species are reported. However 16 Aconitum species are listed in medicinal and aromatic plants database of Nepal (MAPDON). It is commonly known as bikh in Nepal and Monk's hood or aconite in English ${ }^{3}$. The tubers of Aconitum are used as antipyretic and analgesic in the far western Nepal. The tubers are also used for tonsillitis, sore throat, gastritis and debility. In comparing the ethnomedical use with modern 
pharmacological study, it shows consistency with latest pharmacological finding. Caffeic acid of $A$. koreanum is antioxidative and antiinflammatory ${ }^{4}$. In japan, some cases of aconite poisoning appeared as result of committing suicide or accidental ingestion, mistaken for edible grass. However aconite alkaloids have potential of serious and even fatal cardiotoxity, which management has still remained difficult to save in patients with therapeutic resistant fatal arrhythmia ${ }^{5}$. This report details a case of aconite induced ventricular premature complexes (VPC) with right bundle branch block (RBBB) which converted to normal sinus rhythm spontaneously.

\section{Case report}

A 50-year-old lady presented in the emergency unit of B.P. Koirala Institute of Health Sciences, Dharan, Nepal with history of ingestion of herbal plant 'ekfale' (aconite) three hours prior due to family conflict. It was followed by multiple episode of vomiting containing ingested food particles, generalized tingling and burning sensation, restlessness and agitation after one hour. The patient was taken to nearby hospital managed conservatively and was referred to our hospital after half hour of hospital stay for further management. On presentation to our emergency unit patient was conscious but restless and agitated. The patient did not have any medical or surgical problems. There were no known drug allergies. On examination, her vital signs were: blood pressure $90 / 60 \mathrm{mmHg}$, pulse rate $88 \mathrm{bpm}$, respiratory rate $18 /$ minute, temperature afebrile and oxygen saturation $92 \%$ on room air. Chest, cardiovascular system and abdomen examination revealed nothing abnormal. Electrolytes and arterial blood gas report was normal. Both renal and liver function tests results were unremarkable. Electrocardiography revealed multiple ventricle premature complexes with right bundle branch block. The patient was managed with administrations of intravenous fluid and proton pump inhibitor. After receiving bolus of $500 \mathrm{ml}$ normal saline her blood pressure was $110 / 80 \mathrm{~mm}$ of $\mathrm{Hg}$. Internal medicine consultation was done. It was advised to add anti-arrhythmic drug if arrhythmia persisted and she was put on cardiac monitoring. She was symptom-free six hours after consumption of the herbal. Next day early morning, monitor showed no ventricular premature complexes. Twelve-lead electrocardiography showed normal sinus rhythm. The rhythm abnormality spontaneously converted to normal sinus rhythm eight hours later. Patient was admitted to medicine ward with close observation for two days and was discharged after psychiatry consultation as the act was impulsive. 


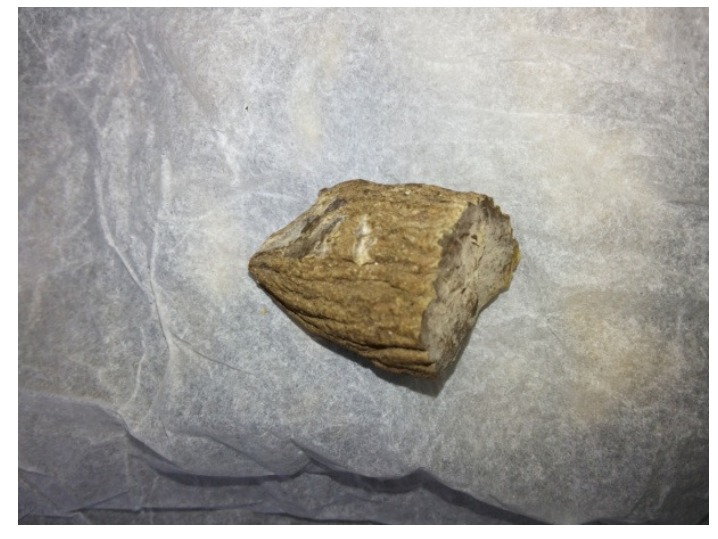

Fig. 1: The remaining herbal root from which the patient ingested

\section{Discussion}

Aconite is well known, because it is extremely toxic. The tuberous root has been used in traditional medicine, although all parts of the plant are considered to be toxic. Extracts of the plant are used in homeopathic and traditional medicine like hypotensives, to decrease fever, as cardiac depressants, and to treat neuralgia ${ }^{6}$. Aconitine and similar alkaloids bind to the open state of voltagesensitive sodium channels in excitable tissue, such as the myocardium, nerves and muscles. Aconitine binds to the sodium channel with high affinity and causes the channel to remain in an active state. This results in continuous depolarisation of the sodium channels, which become refractory to excitation ${ }^{7}$.This continuous state of depolarisation results in both peripheral and central actions. With oral ingestion of aconitine, symptoms of toxicity can appear as soon as 15 minutes to two hours after ingestion $^{8}$. The toxic effects in humans include gastrointestinal symptoms such as nausea, vomiting, diarrhoea and abdominal pain. Neurological features that include paraesthesia, numbness of face and muscle weakness are commonly present $^{8}$. Cardiovascular complications including hypotension, palpitations, ventricular ectopics, ventricular arrhythmias and death may ensue 9 . Our patient had relatively rapid onset of neurological manifestation and cardiotoxicity, although there was no significant gastrointestinal manifestation. She developed typical electrographic changes of frequent PVCs and RBBB. The diagnosis in this case was aided by the identification of aconitine which was brought (Figure 1). There is no antidote for aconitine poisoning and the only treatment at present is supportive care. Ventricular arrhythmias, as in our case, are often refractory to cardioversion and antiarrhythmic drugs. First-line treatments that have been proven to be useful are the use of amiodarone and flecainide $^{7}$. For patients who are in cardiogenic shock and ventricular arrhythmias that do not resolve, a cardiopulmonary bypass may be necessary. The half-life of aconitine has been reported to be about three hours ${ }^{8}$. Various electrocardiographical abnormalities have been identified in aconite herbs poisoning; 
however, ventricle premature complexes with right bundle branch block has rarely been reported previously. Therefore we report this case of reversible right bundle branch block in acute aconite herb poisoning. Physicians should be well-aware with the knowledge and treatment of acute herbal poisoning as it is a real challenge when such cases are encountered.

\section{Conclusion}

Aconitine and its related alkaloids can cause toxic effects and even fatal poisoning. These cases point to the need for strict surveillance of herbal substances. Physicians working in areas where traditional medicine are commonly used must look into these type of patients with high index of suspicion.

\section{References}

1. Mahajani SS, Joshi RS, Gangar VU. Some observations on the toxicity and antipyretic activity of crude and processed aconite roots. Planta Med 1990; 56: 665.

2. Manandhar NP. Plants and People of Nepal. Portland, Oregan, Timber Press Inc, 2002.
3. Shyaula LS Phytochemicals, Traditional uses and processing of Aconitum species in Nepal. Nepal Journal of Science and Technology 2011;12:171-178.

4. Kunwar, RM Shrestha, KP, Bussmann, RW. Traditional herbal medicine in Farwest Nepal: a pharmacological appraisal. J. of Ethno and Ethnomed 2010;6:35.

5. Kolev ST, Leman P, Kite GC, Stevenson PC, Shaw D, Murray V.S. Toxicity following accidental ingestion of Acotinum containing Chinese remedy. Hum Exp Toxicol 1996; 15: 839-842.

6. Spoerke DG. Herbal medications. Santa Barbara, CA: Woodbridge press publishing company; 1980.

7. Chan TY. Aconite poisoning. Clin Toxicol (Phila) 2009; 47:279-85.

8. Moritz F, Compagnon P, Kaliszczak IG, et al. Severe acute poisoning with home made Aconitum napellus capsules: toxicokinetic and clinical data. Clin Toxicol (Phila) 2005; 43:873-6.

9. Fujita $Y$, Terui $K$, Fujita $M$, et al. Five cases of aconite poisoning: toxicokinetics of aconitines. J Anal Toxicol 2007; 31:132-7 